\section{The use of urinary biomarkers to predict the development of azotaemia following treatment of hyperthyroidism in cats}

\section{Henrietta Dillon', Joy Archer', Hattie Syme $^{2}$, Jonathan Elliott ${ }^{2}$, Tim Williams ${ }^{1}$ \\ University of Cambridge, Cambridge, UK \\ 2 Royal Veterinary College, London, UK}

Hyperthyroidism increases glomerular filtration rate and decreases serum creatinine concentrations, which can "mask" underlying azotaemic chronic kidney disease (CKD); thus up to $49 \%$ of cases do not develop azotaemic CKD until after treatment for hyperthyroidism. Albumin and cystatin C in the renal ultrafiltrate are mostly reabsorbed or catabolised by the proximal tubular cells, therefore increased urinary excretion of albumin and cystatin C may correlate with the presence of concurrent CKD.

The aim of this study was to assess the use of urine cystatin C:creatinine ratio (UCC) and urinary albumin:creatinine ratio (UAC) as biomarkers of masked azotaemic CKD in hyperthyroid cats.

Fifty-six hyperthyroid cats were recruited from two first opinion practices. All cats were non-azotaemic at the time of starting treatment for hyperthyroidism. Cats with evidence of other systemic disease were excluded.

Two groups were established according to renal status three months following successful treatment for hyperthyroidism: cats in group A remained non-azotaemic $(n=21)$ and cats in group $B$ developed azotaemia $(n=35)$. A control group of 38 healthy, non-hyperthyroid, non-azotaemic cats was assigned using data gathered from previous studies.

UCC and UAC were determined using previously validated automated immunoturbidimetric assays. Non parametric statistics were used to compare variables of interest between groups at baseline and following treatment. Data are presented as median [25th, 75th percentile].

Hyperthyroid cats $(n=56)$ had significantly higher UAC than control cats $(29.35$ [15.46, 44.89] vs 9.14 [5.0, 13.97]; $\mathrm{P}<0.001)$ and the UAC decreased following treatment for hyperthyroidism (pre-treatment 29.35 [15.46, 44.89] vs posttreatment 15.87 [6.61, 31.05]; $n=30, P=0.045)$. There was no significant difference in UCC between hyperthyroid and control cats $(8.75$ [3.86, 108.3] vs 10.48 [4.87, 19.09]; $P=0.530)$ and the UCC did not change significantly following treatment (pre-treatment 8.75 [3.86, 108.3] vs post-treatment 6.96 [3.86, 20.03]; $n=30, P=0.125)$. There was no significant difference in UCC or UAC between Groups A and B (UCC: Group A 5.6 [4.0, 106.0] vs Group B 10.9 [3.6, 120.2]; $\mathrm{P}=0.855$, UAC: Group A 29.4 [13.6, 46.1] vs Group B 29.4 $[17.5,47.4] ; P=0.555)$.

This study suggests that hyperthyroid cats excrete increased amounts of albumin in their urine which decreases following restoration of euthyroidism. Hyperthyroidism does not appear to affect overall urinary elimination of cystatin C. Neither UCC nor UAC can be used as reliable biomarkers for detection of masked azotaemic CKD in hyperthyroid cats.

\section{Dietary phosphate restriction does not alter urinary active TGF- $\beta 1$ excretion in cats with chronic kidney disease}

\section{Jack Lawson, Harriet Syme, Caroline Wheeler-Jones, Jonathan Elliott}

Royal Veterinary College, London, UK

Chronic kidney disease (CKD) is a common condition of aging cats and the predominant pathological diagnosis is chronic tubulointerstitial inflammation and fibrosis. The cytokine TGF- $\beta 1$ is believed to be an important pro-fibrotic mediator, and urinary active TGF- $\beta 1$ has been shown to correlate with histopathological evidence of renal fibrosis in cats. Severity of interstitial fibrosis is also correlated with plasma phosphate concentration, and feeding of a phosphate restricted diet is a mainstay of therapy for cats with CKD. The aim of this retrospective study was to investigate whether feeding of a phosphate restricted renal diet altered urinary active TGF- $\beta 1$ excretion in cats with CKD.

The records of two London first opinion practices were searched for cats diagnosed with CKD between 2000-2014 with urine samples available prior to and 4-8 weeks after commencing a commercially available phosphate restricted diet. This group ("diet group") was further divided into cats hyperphosphataemic or normophosphataemic for their IRIS stage at baseline. A comparator group was formed of cats diagnosed with CKD which did not accept the phosphate restricted diet. Cats with hyperthyroidism, uncontrolled hypertension or evidence of urinary tract infection were excluded. Urinary active TGF- $\beta 1$ was measured using an ELISA and normalised as a urine TGF- $\beta 1$ : urine creatinine ratio (TGF- $\beta 1: U C r)$. Results are reported as median [25th, 75th percentiles]. TGF- $\beta 1: U C r$ was log-transformed prior to statistical analysis due to non-Gaussian distribution. Effect of dietary phosphate restriction on TGF- $\beta 1: U C r$ was assessed with the two way repeated measures ANOVA.

Forty-one cats were included in the study: 26 in the dietary phosphate restriction group (14 hyperphosphataemic, 12 normophosphataemic) and 15 cats in the comparator group. There was no significant effect of dietary phosphate restriction on TGF- $\beta 1: \mathrm{UCr}$ when comparing cats in the diet group (baseline: 20.4 pg/mg [14.6, 35.3], 4-8 wks: 25.9 pg/ $\mathrm{mg}[9.4,41.8])$ and comparator group (baseline: $16.3 \mathrm{pg} / \mathrm{mg}$ [10.3, 28.8], 4-8 wks: $28.0 \mathrm{pg} / \mathrm{mg}[18.3,33.9])(p=0.98)$ There remained no significant effect when cats in the diet group were subdivided into hyperphosphataemic (baseline: $33.5 \mathrm{pg} / \mathrm{mg}$ [13.0, 34.6], 4-8 wks: 35.99 pg/mg [12.2, 115.0]) and normophosphataemic (baseline: $19.5 \mathrm{pg} / \mathrm{mg}$ [15.7, 25.9], 4-8 wks: $19.1 \mathrm{pg} / \mathrm{mg}[4.6,26.8])(p=0.38)$.

In conclusion, feeding a phosphate restricted diet to cats with chronic kidney disease had no effect on urinary active TGF- $\beta 1$ after 4-8 weeks. Further studies are needed to examine the effects of phosphate restriction on urinary active TGF- $\beta 1$ over the longer term. 